\title{
Propagation of extragalactic cosmic rays in the Galactic magnetic field
}

\author{
Alex Kääpä, ${ }^{a, *}$ Karl-Heinz Kampert $^{a}$ and Eric Mayotte ${ }^{a}$ \\ ${ }^{a}$ Bergische Universität Wuppertal, Gaußstraße 20, 42119 Wuppertal, Germany \\ E-mail: a.kaeaepae@uni-wuppertal.de, kampert@uni-wuppertal.de, \\ mayotte@uni-wuppertal.de
}

The propagation of extragalactic cosmic rays in the Galactic magnetic field plays a crucial role in understanding the cosmic ray signal measured at Earth. This is particularly true for the energy range where the transition from Galactic to extragalactic cosmic rays occurs $\left(E \approx 10^{15.5-18.5} \mathrm{eV}\right)$. The reason for this is that the Galactic magnetic field strength is such that cosmic ray propagation in this energy range will change from diffusive to ballistic and is thus central to understanding the exact nature of the source transition.

Using simulation studies with CRPropa3, we study the effects that propagation in the Galactic magnetic field will imprint on cosmic rays in the rigidity range $10^{16-20} \mathrm{~V}$ for both isotropically and anisotropically injected extragalactic cosmic rays. As a result, in case of isotropic injection we find that the Galactic magnetic field neither modifies the flux nor the arrival direction distribution across the entire rigidity range. For injection of dipole-like flux anisotropies as well as for single point sources, we find flux modifications across the entire rigidity range which depend on the direction and nature of the anisotropy. We also find that the arrival direction distribution is consistent with isotropy below rigidites of $10^{18} \mathrm{~V}$, and the remaining anisotropy for all particles integrated above rigidities of $10^{18} \mathrm{~V}$ manifests in the form of dipoles at the 1-10\%-level.

\footnotetext{
*** The European Physical Society Conference on High Energy Physics (EPS-HEP2021), ***

*** 26-30 July $2021 * * *$

*** Online conference, jointly organized by Universität Hamburg and the research center DESY ***
}

\footnotetext{
${ }^{*}$ Speaker
} 


\section{Introduction}

Extragalactic cosmic rays (EGCRs) are thought to dominate the flux of cosmic rays above the so-called "ankle" at around $3 \cdot 10^{18} \mathrm{eV}[1,2]$. Below the ankle, EGCRs are expected to contribute significantly to the all-particle flux as the flux of Galactic cosmic rays (GCRs) is suppressed. It is not clear, where this transition from GCRs to EGCRs occurs however. A key aspect to consider in understanding the transition region is the effect of the Galactic magnetic field (GMF) on CR observables $^{1}$. The reason for this is that this energy range also signifies a change in propagation regimes from diffusive to ballistic. It has already been shown using simulations with two GMF models that the propagation of CRs on average becomes ballistic at a rigidity ${ }^{2}$ of $\approx 6 \mathrm{EV}[3]$. A qualitative understanding of this change in propagation regimes can be gained with the help of the gyroradius $^{3}$. In Fig. 1, the gyroradius $r_{g}$ as a function of rigidity $R$ for typical GMF strengths is depicted. Two important Galactic length scales, the width and radius of the Galactic plane (GP), $h_{\mathrm{GP}}\left(\approx\right.$ a few kpc) and $r_{\mathrm{GP}}(\approx 10-20 \mathrm{kpc})$, respectively, as well as the approximate coherence length $\lambda(\lesssim 100 \mathrm{pc})$ of the random component of the GMF $[6]$ are added as horizontal lines.

At rigidities of a few $\mathrm{EV}, r_{g}$ approaches $r_{\mathrm{GP}}$, and $\mathrm{CR}$ propagation can be characterised as ballistic. At rigidities where $r_{g}<\lambda$, CRs propagate diffusively. This change in propagation regimes may plausibly affect the flux of EGCRs. With decreasing rigidity, they will be increasingly shielded from the Galaxy, while those that do reach the GP will be confined therein. This results in a rigiditydependent shift of the direction of increased or decreased GMF transparency to EGCRs. While Liouville's theorem predicts the conservation of flux and isotropy in the case of isotropic injection [7], mod-

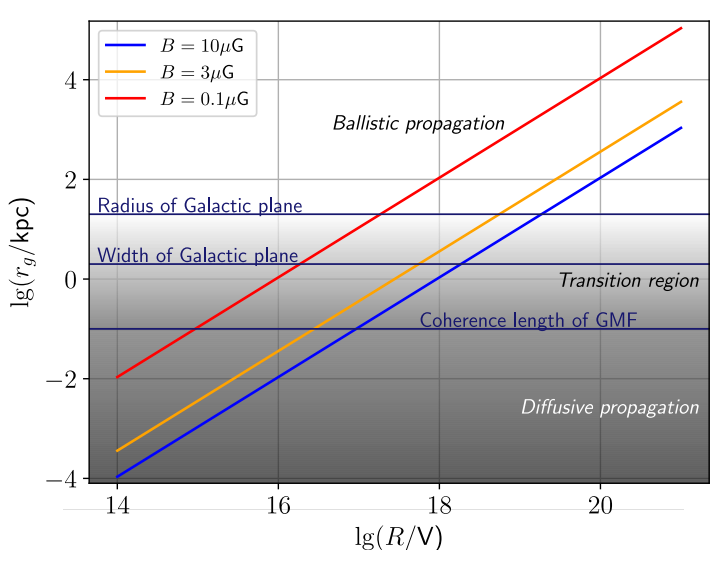

Figure 1: Gyroradius $r_{g}$ as a function of rigidity $R$ for typical GMF strengths. Horizontal lines signify typical Galactic length scales, as well as the coherence length of the random component of the GMF. ifications in flux and arrival direction may arise if EGCRs are injected anisotropically.

We study the propagation effects imposed by the GMF via Monte-Carlo simulations and start by performing forward-tracking studies of isotropically injected EGCRs. This allows for investigating the change of EGCR densities in the Galaxy, and we may confirm Liouville's theorem. Later on, anisotropic injections of EGCRs are studied using the Galactic lensing scheme [3, 8]. Only proton primaries are simulated as only deflections in the GMF are studied and interactions are neglected meaning all effects are rigidity-dependent ${ }^{4}$.

\footnotetext{
${ }^{1}$ Indeed, while studies of the impact of the GMF on the arrival direction distribution have been performed for EGCRs at energies around the "ankle" and higher [3-5], the effect on both flux and composition of CRs has sofar not been fully considered.

${ }^{2}$ The rigidity $R$ is related to the energy of a CR particle via $E=R \cdot Z e$, where $Z e$ is the nuclear charge of the CR.

${ }^{3}$ The gyroradius $r_{g}$ provides the strength of deflection of particle with rigidity $R$ in a magnetic field of strength $B$ via $r_{g} \approx 11 \frac{R[\mathrm{PV}] \cdot \beta_{\perp}}{B[\mu \mathrm{G}]} \mathrm{pc}$, where $\beta_{\perp}=\frac{v_{\perp}}{c}$.

${ }^{4}$ Results for other nuclear species can be retrieved via appropriate scaling with the corresponding charge number.
} 


\section{Simulation setup}

The simulation studies are performed with CRPropa3 [9]. We employ the ballistic propagation module where individual particles are tracked by solving the equation of motion. The JF12 model [6] is used for the GMF, since it is currently the most commonly used. All three components, the regular, striated (large-scale random) and (small-scale) random component, are included. The random seed that sets the latter two components is reset for each particle. Two kill conditions for the simulation are set. The first is the edge of the Galaxy (i.e. the edge of the GMF), which is a $20 \mathrm{kpc}$ shell (henceforth referred to as the Galactic shell) around the Galactic centre (GC) for the JF12 model. The second condition is a maximum trajectory length of $1 \mathrm{Gpc}$.

For our forward-tracking studies, the source of EGCRs mimics that of isotropic injection via a uniform source density along the Galactic shell with a Lambertian injection direction distribution. There are two defined observers. The first one is the GP itself, $O_{\mathrm{GP}}$, parameterised via a cylinder of $100 \mathrm{pc}$ thickness centered around the GC. The radius was set to $19.5 \mathrm{kpc}<\mathrm{r}_{\mathrm{GP}}$ to account for a systematic bias arising from the discontinuous drop-off of the field strength at the edge of the Galaxy for the JF12 field ${ }^{5}$. The second observer is Earth, $O_{\mathrm{E}}$, parameterised as a shell centered at the location of Earth in Galactic coordinates $(x=-8.5 \mathrm{kpc})$. The radius of the shell is varied between $5 \mathrm{pc}$ and $1 \mathrm{kpc}$ in order to identify artefacts stemming from the nonzero observer size.

The rigidity range of $10^{16-20} \mathrm{~V}$ is set for the simulated particles. For lower rigidities, simulation of a large number of particles becomes computationally unfeasible with the available resources ${ }^{6}$. However, the flux of EGCRs at these rigidities is expected to be subdominant. Finally, all CRs are injected with an $R^{-1}$-spectrum, to make possible modifications in the spectrum visually discernible.

\section{Propagation effects - Confinement and shielding}

At high rigidities where propagation can be considered ballistic, the fraction of EGCRs that reach an observer can be inferred from the field-free case. As the rigidity falls below the characteristic length scales of the GP, the effect of the GMF starts to play a role. It increasingly shields EGCRs from the GP, while trapping or confining those that do reach the GP. We thereby have two counteracting effects for EGCRs, both of which increase as rigidity goes down. The conservation of flux and isotropy for isotropic injection predicted by Liouville's theorem implies that confinement and shielding exactly cancel, and that the GMF does not introduce any intrinsic anisotropy. This we seek to verify, as it not only substantiates the proposed simulation approach, but also lays the necessary foundation for the subsequent Galactic lensing scheme. In case of an anisotropic injection, the effects of the GMF are more subtle. The shifting direction of GMF transparency may lead to flux modifications depending on the nature of the anisotropy. The arrival direction distribution of EGCRs is expected to be increasingly smeared out as rigidity decreases. Therefore, the injection of anisotropy can be viewed as a test of the degree of isotropisation by the GMF.

\footnotetext{
${ }^{5}$ This discontinuity enables low-rigidity particles to penetrate further into the Galaxy than they would in the case of a continuous drop-off of the field strength beyond the edge of the Galaxy. The surplus particles are eventually reflected back outside the Galaxy. Therefore, reducing the radius functions as a fiducial cut to recover the behaviour which would occur for a continuous drop-off of the GMF.

${ }^{6}$ For the study of the large-scale propagation effects in [3], the relatively large observer size (the GP) allowed for an extension of the rigidity range downward by an order of magnitude to $10^{15-20} \mathrm{~V}$.
} 


\subsection{Quantification of propagation effects}

We consider the relative residence time, $t_{\text {rel }}=t_{\text {in }} / t_{\text {tot }}$, of CRs in the GP to parameterise the confinement therein. Here, the residence time of each CR within the GP, $t_{\text {in }}$, is normalised to its total residence time, $t_{\text {tot }}$, in the Galaxy. This quantity is expected to increase with increasing confinement, and vice versa. The median value of $t_{\text {rel }}$ as a function of rigidity is plotted in Fig. 2a. As expected, we find that confinement is high at lowest rigidities with CRs spending the majority of their total time in the Galaxy within the GP. $t_{\text {rel }}$ decreases rapidly above around $0.1 \mathrm{EV}$ and flattens out above a few EV, where the corresponding gyroradius exceeds the characteristic length scales of the Galaxy.

The shielding of EGCRs from the GP is parameterised through the number of CRs reaching the $\mathrm{GP}, N_{\mathrm{CR}, \mathrm{GP}}$. This is depicted in Fig. $2 \mathrm{~b}$ as a function of rigidity. As expected, the count increases with rigidity. The trend becomes steeper until around $10^{17} \mathrm{~V}$, and finally saturates at a few EV.

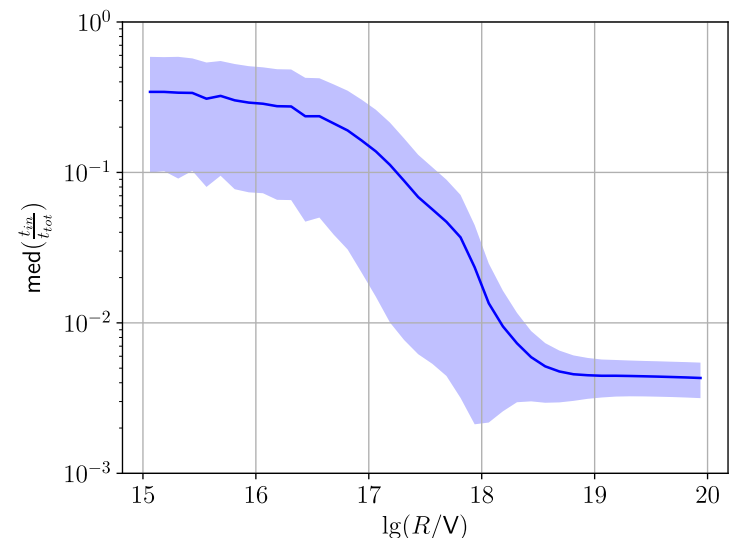

(a) Median (lines) and the median absolute distance (shaded areas) of $t_{\text {rel }}$ as a function of rigidity.

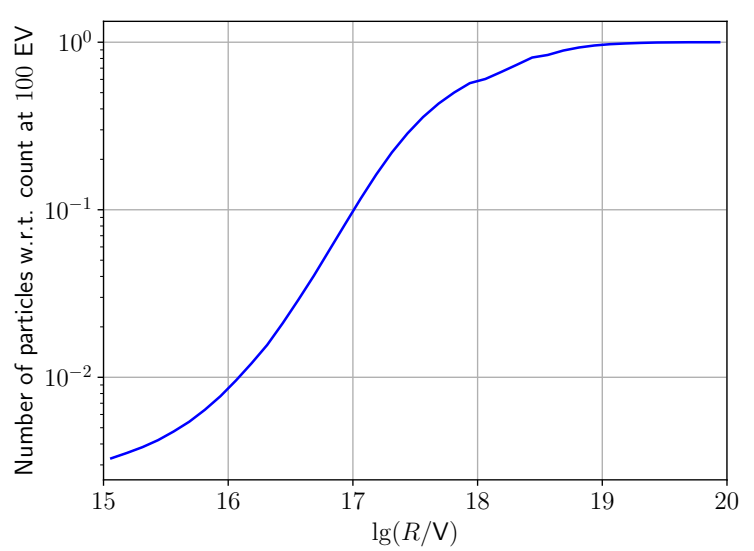

(b) The total number of EGCRs reaching the GP as a function of rigidity.

Figure 2: Quantification of the confinement of GCRs and EGCRs.

\section{Effects on flux, composition and arrival direction}

After having identified the large-scale propagation effects (confinement and shielding), we now study how they manifest for observables such as flux, composition, and arrival directions. We forward track isotropically injected EGCRs from their respective sources to Earth and calculate the flux and rigidity spectrum of EGCRs via the number of CRs which cross $O_{\mathrm{E}}$. This is then normalised to the expected count from the field-free case. The rigidity spectrum for various shell radii is depicted in Fig. 3. While there seems to be a flux suppression towards smaller rigidities for larger sphere sizes, this suppression appears to vanish as the observer approaches a point (i.e. where $r_{\mathrm{obs}} \rightarrow 0$ ). This confirms that shielding and confinement cancel exactly for isotropically injected EGCRs. We also find an isotropic arrival direction distribution at Earth across the entire rigidity range, meaning that the GMF does not introduce any intrinsic anisotropy. 
As previously mentioned, the effects of an injected anisotropy are investigated via the lensing method. In it deflections in the GMF are quantified via a matrix, the lens, which associates an arrival direction distribution for any given injection direction distribution. The lens was created by backtracking anti-particles from the position of the observer to the edge of Galaxy, where the source is situated, and subsequently inverting the directions. This was then tested by confirming that it yields an isotropic arrival direction distribution and a conserved flux in the case of isotropic injection.

We investigated two different (pure) anisotropy scenarios. The first was that of a dipole, the largest-scale anisotropy that may be measured. The second was that of a point source, the strongest type of anisotropy that may be injected. We injected the dipole from the directions related to those of highest and lowest GMF transparency, while the point sources were additionally injected from directions of nearby sources, such as Centaurus A [10].

For all injected anisotropies, we found

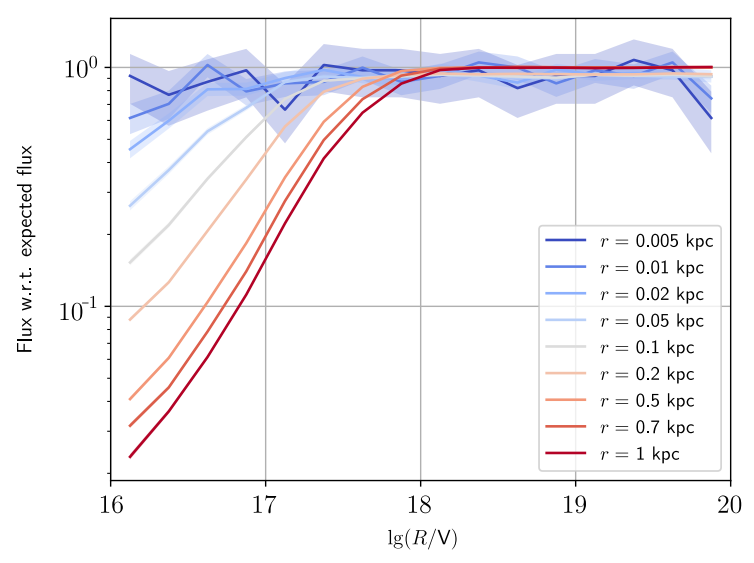

Figure 3: Measured rigidity spectra of EGCRs for various observer shell radii.

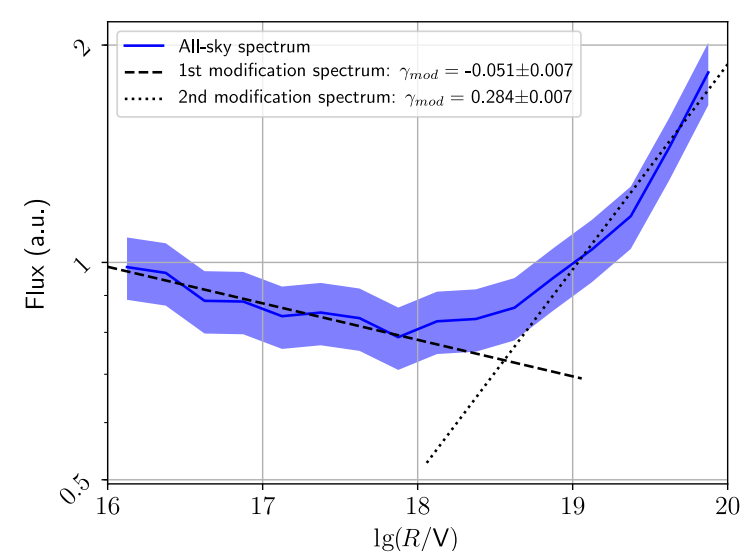

Figure 4: Rigidity spectrum of the lensed distribution from the direction of Centaurus A. a strong isotropisation below roughly $1 \mathrm{EV}$ down to the percent level. At higher rigidities, the only significant anisotropy that remained was that of a dipole for all injected anisotropies. Point sources generated amplitudes of around $10 \%$, as opposed to a few percent for the injected dipoles.

For both the injected dipoles and point sources, a spectral modification was observed. For the injected dipoles, this modification manifested in the form of a change in the spectral index across the entire rigidity range, while for the point sources, a spectral break arose at rigidities of a few EV for some directions. The rigidity spectrum of the lensed distribution from the direction of Centaurus A is depicted in Fig. 4, where the spectral break appears even "ankle"-like.

\section{Conclusion}

The presented work strongly suggests that the effects of CR propagation in the GMF on the spectrum, composition and arrival directions need to be taken into account in the energy range signifying the transition from GCRs to EGCRs. We showed that this can be tied to the fact that the transition in propagation regimes from diffusive to ballistic also occurs in this energy range. The effects are rather subtle stemming from the directional shift in the GMF transparency. 
Only for anisotropic injection are EGCR observables affected. We found a strong isotropisation by the GMF. An injected dipole can survive only above a rigidity of $R \approx 1 \mathrm{EV}$ with an amplitude observed at the 1-10\%-level, depending on the nature of the dipole. More strikingly, the propagation in the GMF leads to a spectral modification, the nature and strength of which depend on the nature and direction of the injected anisotropy. An injected dipole leads to a smoother change with only a modification of the spectral index, while injection from a point source leads to spectral breaks in the rigidity range of a few EV. Especially noteworthy is that the injection from the direction of Centaurus A leads to a hardening of the spectrum, very similar in location and shape to that of the "ankle". This may partly tie the occurrence of the "ankle" to propagation effects in the GMF. However, given the fact that more realistic EGCR fluxes contain both an isotropic and anisotropic component, the measured anisotropy and flux modification due to propagation in the GMF are expected to be weaker than found here. To describe the measured spectral, composition and anisotropy data fully, additional assumptions about the injected spectrum need to be made, as changes from the GMF alone cannot account for them. In general, the sum of the GCR and EGCR contributions needs to be considered to account for the observed features in the transition region.

Our work can be expanded upon most concretely by making more realistic assumptions about the source distribution and spectra. Gauging the degree of expected anisotropy of EGCRs injected into the Galaxy is of special importance for understanding the effect that propagation in the GMF may have on CRs.

Acknowledgments: The simulations were carried out on the Pleiades cluster at the University of Wuppertal, which was supported by the Deutsche Forschungsgemeinschaft (DFG). Further financial support by the German Ministry of Research and Education (Verbundforschung Astroteilchenphysik) is gratefully acknowledged.

\section{References}

[1] A. Aab et al., [Pierre Auger Coll.], Phys. Rev. Lett. 125 (2020) 121106 [2008. 06488].

[2] A. Aab et al., [Pierre Auger Coll.], Science 357 (2017) 1266 [1709.07321].

[3] M. Erdmann, G. Müller, M. Urban and M. Wirtz, Astropart. Phys. 85 (2016) 54 [1607.01645].

[4] G.R. Farrar and M.S. Sutherland, JCAP 05 (2019) 004 [1711. 02730].

[5] B. Eichmann and T. Winchen, JCAP 04 (2020) 047 [2001.01530].

[6] R. Jansson and G.R. Farrar, ApJ 757 (2012) 14 [1204. 3662].

[7] T.K. Gaisser, R. Engel and E. Resconi, Cosmic Rays and Particle Physics: 2nd Edition, Cambridge University Press $(6,2016)$.

[8] H.-P. Bretz, M. Erdmann, P. Schiffer, D. Walz and T. Winchen, Astropart. Phys. 54 (2014) 110 [1302 . 3761].

[9] R. Alves Batista, A. Dundovic, M. Erdmann, K.-H. Kampert, D. Kuempel, G. Müller et al., JCAP 05 (2016) 038 [1603.07142].

[10] A. Aab et al., [Pierre Auger Coll.], Astrophys. J. Lett. 853 (2018) L29 [1801. 06160]. 